\title{
Distance-Dependent Measurements of the Conductance of Porphyrin Nanorods Studied with Conductive Probe Atomic Force Microscopy
}

\author{
Xianglin Zhai ${ }^{\dagger}$, Denzel Alexander ${ }^{\dagger}$, Pedro Derosa ${ }^{\ddagger}$, and Jayne C. Garno ${ }^{*} \dagger$ \\ †Department of Chemistry, Louisiana State University, Baton Rouge, Louisiana 70803, United \\ States \\ FInstitute for Micromanufacturing, Chemistry Department, and Physics Department, Louisiana \\ Tech University, Ruston, Louisiana 71272, United States
}

\section{Abstract}

Protocols for nanopatterning porphyrins on $\mathrm{Au}(111)$ were developed based on immersion particle lithography. Porphyrins with and without a central metal ion, 5,10,15,20-tetraphenyl-21 H,23 Hporphyrin (TPP) and 5,10,15,20-tetraphenyl-21 H,23 H-porphyrin cobalt(II) (CoTPP), were selected for study, which spontaneously formed nanorod geometries depending on concentration parameters. The elongated shapes of the nanorods offers an opportunity for successive distancedependent conductive probe atomic force microscopy (CP-AFM) measurements along the length of the nanorods. To prepare patterns of TPP and CoTPP nanorods, a mask of silica mesospheres was placed on gold substrates to generate nanoholes within an alkanethiol matrix film. The nanoholes prepared by particle lithography with an immersion step were backfilled with porphyrins by a second immersion step. By controlling the concentration and immersion interval, nanorods of porphyrins were generated with one end of the nanostructure attached to gold within a nanohole. The porphyrin nanorods exhibited slight differences in dimensions at the nanoscale to enable size-dependent measurements of conductive properties. The conductivity along the horizontal direction of the nanorods was evaluated with CP-AFM studies. Changes in conductivity were measured along the long axis of TPP and CoTPP nanorods. The TPP nanorods exhibited conductive profiles of an insulating material, and the CoTPP nanorods exhibited profiles of a semiconductor. The experiments demonstrate the applicability of particle lithography for preparing unique and functional surface platforms of porphyrins to measure distance-dependent conductive properties on gold.

\section{Graphical Abstract}

*Corresponding Author: (J.C.G.) Phone 225-578-8942; jgarno@1su.edu.

ORCID

Jayne C. Garno: 0000-0003-3446-1780

The authors declare no competing financial interest.

Supporting Information

The Supporting Information is available free of charge on the ACS Publications website at DOI: 10.1021/acs.langmuir.6b03525. Further experimental details, results of the control experiments, surface morphology of CoTPP nanorods, and the dimensions of the nanorods (PDF) 


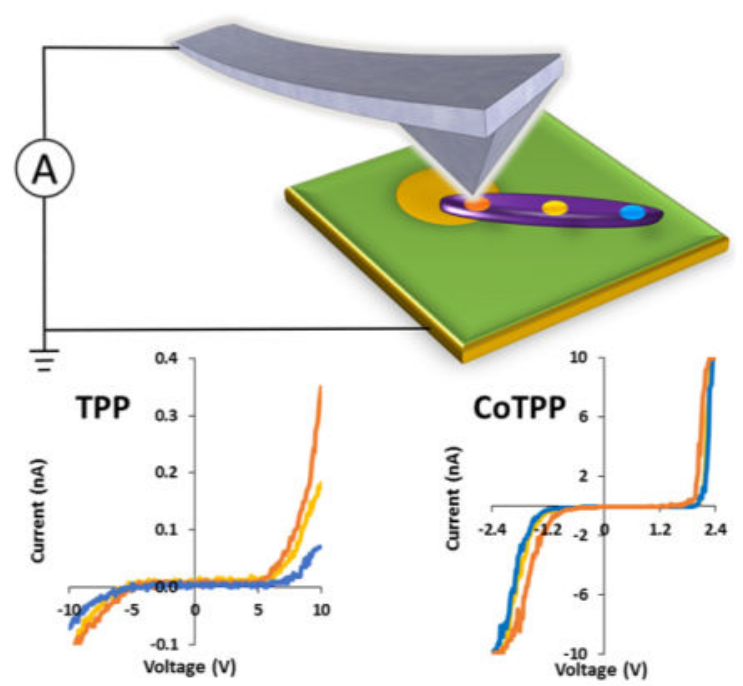

\section{INTRODUCTION}

Porphyrin molecules and associated derivatives are building blocks in molecular electronics, photovoltaic materials, and supramolecular designs. ${ }^{1}$ Nanorods of porphyrins have potential for applications including energy and electron transfer, ${ }^{2,3}$ chemical catalysis, ${ }^{4}$ chemical sensors, ${ }^{5}$ optical devices, ${ }^{6}$ electronic devices, ${ }^{3}$ biological light-harvesting structures, ${ }^{3}$ gas storage, field-effect devices, ${ }^{3}$ and chiroptical systems. ${ }^{5}$ The applications originate from the unique characteristics of porphyrins that include rigid and planar geometries, readily tailored spectroscopic and photochemical properties, multifunctionality, biocompatibility, ${ }^{5}$ and the ability to serve as an electron donor. ${ }^{2}$ The conductive properties of porphyrins and derived nanostructures have not been fully characterized or modeled.

Methods of surface fabrication with porphyrin nanorods include surfactant-assisted selfassembly, ${ }^{3}$ ionic self-assembly, ${ }^{7,8}$ mixed porphyrin self-assembly, ${ }^{9}$ sonication-assisted selfassembly, ${ }^{2}$ and metal coordination-assisted self-assembly. Among the studies reported for porphyrin nanorods, rodlike aggregates derived from meso-tetra(4sulfonatophenyl)porphyrin ( $\mathrm{TPPS}_{4}$ ) are a well-studied example because it is a biomimetic analogue of photosynthesis systems. ${ }^{710-20}$ The heights and lengths of the $\mathrm{TPPS}_{4}$ nanorods range from several to dozens of nanometers in diameter and up to several micrometers in length. ${ }^{4,10,13,19,20}$ The size and shapes of the nanorods derived from TPPS $_{4}$ are due to a combination of molecular interactions such as Coulombic interactions, $\pi-\pi$ interactions, and intermolecular electrostatics. ${ }^{21}$ Studies were reported by Schuckman et al. of the charge transport properties of a tethered $\mathrm{Zn}$ (II) porphyrin thiol complex, tripyridyl porphyrin, in which molecular clusters were inserted within an alkanethiol matrix on $\mathrm{Au}(111){ }^{22,23}$

For this report, a self-assembled monolayer (SAM) of dodecanethiol on Au(111) was selected as an insulating layer between the porphyrin nanorods and the gold substrate for CP-AFM measurements. ${ }^{24-26}$ A surface template of nanoholes was fabricated within a dodecanethiol SAM using a method of immersion particle lithography. ${ }^{27}$ We have developed protocols with immersion particle lithography to prepare arrays of nanoholes within organic 
thin films on surfaces such as gold, mica, and silicon. ${ }^{27-31}$ Typically, when a surface that is coated with mesoparticles is immersed in solutions, the particles are displaced. Therefore, an annealing step is critical for immersion particle lithography. The nanoholes formed within a SAM furnish spatially confined areas for growing porphyrin nanorods with one end connected to the gold surface. The surface-bound patterns provide an excellent measuring platform for evaluating conductivity along the length of porphyrin nanorods. Previously, we prepared samples of smaller nanodot structures of 5,10,15,20-tetraphenyl-21 $\mathrm{H}, 23 \mathrm{H}$ porphyrin (TPP) and 5,10,15,20-tetraphenyl-21 H,23H-porphyrin cobalt(II) (CoTPP) porphyrins by using lower concentrations and immersion intervals. ${ }^{32}$ The measured conductivity was substantially different for the longer nanorods compared to the nanodot samples. To account for the differences in measurements for size and shape, further studies with modeling the intermolecular interactions are in progress.

In this report, the feasibility of making distance-dependent conductance measurements along the length of isolated nanorods is evaluated with CP-AFM for test platforms of TPP and CoTPP nanorods. The elongated, rod shape of the porphyin nanorods furnishes a unique platform for nanoscale studies of changes in conductance. The rods are composed of stacks of planar porphyrins with or without a coordinated metal (cobalt) which form spontaneously depending on the growth conditions (immersion interval, concentration). Discrete, isolated areas of the patterned gold substrate provided a location for the nanorods to assemble. A matrix layer of dodecanethiol serves to separate the sites for the nanorods to attach as well as to provide an internal reference for conductive probe measurements. Beyond constructing the platforms, conductivity of a cobalt-coordinated and free-base porphyrin were compared.

\section{MATERIALS AND METHODS}

\section{Materials}

Reagents including 5,10,15,20-tetraphenyl-21 H,23H-porphyrin cobalt(II) (CoTPP), 5,10,15,20-tetraphenyl-21 $H, 23 H$-por-phyrin (TPP), 1-dodecanethiol, and dichloromethane were purchased from Sigma-Aldrich (St. Louis, MO). Ethanol (200 proof) was obtained from Pharmco-Aaper Alcohol and Chemical Co. (Shelbyville, KY). Epoxy was acquired from Epoxy Technology, Inc. (Billerica, MA). Gold slugs (99.99\%) were purchased from Ted Pella (Redding, CA). Ruby muscovite mica was obtained from S\&J Trading Inc., (Glen Oaks, NY). Silica mesospheres (500 nm diameter) were obtained from Thermo-Fisher Scientific Inc. (Waltman, MA). Water ( $\geq 18.2 \mathrm{M} \Omega$ ) was generated from a Milli-Q system (Direct-Q 3, Millipore, Billerica, MA) and used for washing. All chemicals were used as received.

\section{Preparation of Porphyrin Solutions}

To prepare porphyrin solutions for making nanostructures on $\mathrm{Au}(111)$ substrates, CoTPP and TPP were dissolved in dichloromethane $\left(10^{-3} \mathrm{M}\right)$ and then further diluted to $10^{-5} \mathrm{M}$ with ethanol. Nanorods of the porphyrins were made by immersing the $\mathrm{Au}(111)$ substrates into final solutions for $42 \mathrm{~h}$. 


\section{Preparation of Template-Stripped Au(111)}

Template-stripped gold (TSG) films were prepared using thermal evaporation as previously described. ${ }^{27}$ In brief, gold slugs were deposited onto freshly cleaved Ruby muscovite mica substrates in a high-vacuum thermal chamber (Angstrom Engineering Inc., Kitchener, OR) at $10^{-7} \mathrm{mmHg}$. Prior to gold deposition, the mica substrates were preheated to $350{ }^{\circ} \mathrm{C}$ using quartz lamps mounted at the back of the sample holder. The deposition rate of gold was 3 $\AA / \mathrm{s}$ for a final thickness of $150 \mathrm{~nm}$. After deposition, the gold substrates were annealed at $365^{\circ} \mathrm{C}$ under vacuum of $10^{-7} \mathrm{mmHg}$ for $30 \mathrm{~min}$ and then cooled to room temperature. The gold films were glued to glass slides using epoxy as previously reported by Hegner et al. ${ }^{33}$ The gold substrates and glass slides were cleaned by rinsing with deionized water followed by $30 \mathrm{~min}$ treatment in a UV-ozone generator. Epoxy was mixed (1:1 by weight) and immediately applied onto the cleaned glass slides. The glass slides were placed onto the gold surfaces with a thin layer of epoxy formed between the glass and gold surfaces without any air bubbles. The mica-gold-glass sandwiches were then heated in oven at $150{ }^{\circ} \mathrm{C}$ for $2 \mathrm{~h}$ to anneal the epoxy. After cooling to room temperature, the glass pieces were mechanically removed from the mica surfaces to obtain TSG.

\section{Preparation of Porphyrin Nanostructures within Thiol Nanoholes}

Immersion particle lithography was used to prepare arrays of nanoholes on $\mathrm{Au}(111)$ as previously reported. ${ }^{27}$ Silica mesospheres (500 $\mathrm{nm}$ diameter) were cleaned by centrifugation to remove surfactants or charge stabilizers. A $300 \mu \mathrm{L}$ aqueous suspension of silica mesospheres with a concentration of $2 \%$ was transferred into a microcentrifuge tube and centrifuged for $20 \mathrm{~min}$ at $20000 \mathrm{rpm}$. A solid pellet containing the spheres was formed at the bottom of the centrifuge tube, and the supernatant was decanted. The centrifuge tube was refilled with deionized water, and the pellet was resuspended by vortex mixing. The washing cycle of mesospheres was repeated four times. Cleaned silica mesospheres $(20 \mu \mathrm{L})$ were deposited onto freshly stripped TSG as surface masks for subsequent steps of solution immersion. The surface masks of silica spheres were dried under ambient conditions and then heated at $150{ }^{\circ} \mathrm{C}$ for $12 \mathrm{~h}$. The heating step helps prevent the mesospheres from being displaced from the surface during immersion in solutions. The samples were cooled to room temperature $\left(25^{\circ} \mathrm{C}\right)$ and then immersed into a dodecanethiol ethanolic solution $\left(10^{-3} \mathrm{M}\right)$ for $24 \mathrm{~h}$. Self-assembled monolayers (SAMs) of dodecanethiol formed on the exposed areas of the gold surfaces surrounding the areas protected by the mesospheres. Next, the mesospheres were removed by sonication in ethanol. Arrays of nanoholes were fabricated within the dodecanethiol SAM.

The samples of nanoholes formed on $\mathrm{Au}(111)$ were submerged in a $10^{-7} \mathrm{M}$ solution of CoTPP or TPP for $42 \mathrm{~h}$ to obtain porphyrin nanorods. In the final step, the samples of porphyrin nanostructures were rinsed with ethanol to remove loosely bound aggregates and then dried in air. The uncovered areas of $\mathrm{Au}(111)$ that had been masked by mesospheres provided well-defined surface sites for directing the subsequent attachment and growth of porphyrin nanorods. 


\section{Atomic Force Microscopy}

A Keysight 5420 scanning probe microscope (Keysight Technologies, Santa Rosa, CA) with PicoView v1.12 software was used for scanning probe characterizations. Images were acquired using tapping mode in ambient conditions. Imaging was accomplished with rectangular-shaped ultrasharp silicon tips (Nanoscience Instruments, Phoenix, AZ) that have an aluminum reflex coating, with a spring constant of $48 \mathrm{~N} / \mathrm{m}$. The scan rate was 1 line/s. Electrical measurements with porphyrins were done with the same instrument equipped with conductive nose assembly ( $0.1 \mathrm{nA} / \mathrm{V}$ sensitivity) and conductive sample plate. Conductive tips (PPP-CONTPt, Nanosensors, Neuchatel, Switzerland) with a platinum coating were used to take the $I-V$ profiles. Topography and phase images were processed with Gwyddion v. 2.32, which is an open-source software available from the Internet. ${ }^{34}$

\section{RESULTS AND DISCUSSION}

Nanorods of porphyrins were prepared on a template of nanoholes within a dodecanethiol film on $\mathrm{Au}(111)$ using steps of immersion particle lithography. A description of the method for preparing patterns of nanorods is outlined in the Supporting Information, Figure S1. Model structures of 5,10,15,20-tetraphenyl-21 $H, 23 H$-porphyrin cobalt(II) (CoTPP) and the metal-free analogue 5,10,15,20-tetraphenyl-21 $\mathrm{H}, 23 \mathrm{H}$-porphyrin (TPP) were selected for studies with CP-AFM. The structures of TPP and CoTPP are shown in Scheme 1.

The morphology of the nanorods formed by either TPP or CoTPP was characterized by tapping mode AFM in ambient conditions (Figure 1). Individual porphyrin nanorods are visible throughout the surface, predominantly binding at sites of the gold nanoholes. Characteristics of the gold surface, such as domain boundaries, scars and holes, are apparent in the background of the topography images (Figures 1a and 1c). The periodic arrangement of nanoholes can be discerned as bright circular dots in the corresponding phase images (Figures $1 \mathrm{~b}$ and 1d). The nanoholes are uncovered areas of substrate where the silica mesospheres were displaced. The distance between neighboring nanoholes is $500 \mathrm{~nm}$ as determined by the diameter of the monodisperse spheres used as surface mask. The nanoholes were protected from deposition of dodecanethiol by the mesospheres, and areas surrounding the nanoholes present methyl groups of the dodecanethiol SAM that was used as a resist. The nanoholes are exquisitely small ( $100 \mathrm{~nm}$ in diameter and $1.5 \mathrm{~nm}$ in depth) and are designed to define spatial selectivity for depositing porphyrins. ${ }^{35,36}$

In most of the nanoholes, one or more nanorods are present and connected to an area of bare gold. A few of the nanorods are present on the SAM matrix areas, which most likely grow in defect areas. Closer details of the nanorods are revealed in Figures 1c and 1d. Among the seven nanoholes, five of them contain TPP nanorods. Two of the nanoholes are unfilled, and four free nanorods are lying on areas of the SAM. We hypothesize that the nanorods of porphyrins were directly grown at the areas of bare $\mathrm{Au}(111)$ during the immersion process, since shorter immersion times lead to shorter nanostructures of nanodots. ${ }^{32}$ To evaluate if nanorods formed in solution, a drop of similarly aged TPP solution was deposited onto a gold surface. Nanorods did not form on the surface (Figure S2). The drop-deposition experiment indicates that the nanorods were not formed in solution during the immersion step, but rather that the rodlike shapes grew from the surface over a time period of $42 \mathrm{~h}$. 
The nanopatterning process for preparing nanorods was repeated with CoTPP using the same concentration of the porphyrin solution and $42 \mathrm{~h}$ immersion period. Rod-shaped nanostructures of CoTPP were also reproducibly grown from the gold surface (Figure S3). The dimensions of a CoTPP nanorod can be derived from cursor profiles taken in the longitudinal and orthogonal directions. The size distribution of the nanorods were compared for TPP and CoTPP (Figure S4). The dimensions of the TPP nanorods were measured from 64 cursor profiles from several areas of a sample. The average length of the TPP nanorods is $160 \pm 41 \mathrm{~nm}$. The average height of the TPP nanorods measured $6 \pm 1 \mathrm{~nm}$. The dimensions of CoTPP nanorods were collected from 82 measurements from several areas of a sample. The CoTPP nanorods measured $130 \pm 49 \mathrm{~nm}$, with heights of $3 \pm 1 \mathrm{~nm}$. The shape of AFM probes influences the sizes that were measured, particularly for lateral dimensions. Sharper probes produce more accurate size measurements.

A representative example image of a TPP nanorod is shown in Figure 2. The nanorod has one end connected to the area of a nanohole. The rest of the nanorod is lying on the dodecanethiol SAM which serves as an insulating matrix. The placement of the nanorod within the nanohole is also visible in the corresponding phase image (Figure 2b). The size of the individual nanorod from the cursor line profiles indicates a length of $160 \mathrm{~nm}$ and height of $6.6 \mathrm{~nm}$.

The conductivity along the length of the nanorods was measured using CP-AFM. A platinum-coated AFM tip was placed at selected points along the nanorod with a controlled load to measure the distance-dependent current. Three testing points were selected along the nanorod to collect the $I-V$ curves (Figure $3 a$ ). The points selected include each end of the porphyrin where one end is in contact with the matrix SAM, and the other is in direct contact with the gold substrate. One test point was located between the two ends. At each of the three sites the voltage was swept from -10 to $+10 \mathrm{~V}$, and the resulting current was measured (Figure 3b).

Trends of the conductive properties correspond to the testing positions. Current-voltage curves indicate an increase in conductance as the tip was moved closer toward the $\mathrm{Au}$ surface. The current at $10 \mathrm{~V}$ increased along the horizontal direction of the TPP nanorod. In reverse, the conductance of the nanorod decreased as a function of the testing distance. The highest current at $-10 \mathrm{~V}$ remained the same during this experiment. The onset of the turn-on current occurs at a lower voltage as the tip is moved closer to the end of the TPP nanorod that is attached to gold.

Similar measurements were done for a cobalt-coordinated CoTPP nanorod localized on a nanopore. The morphology of an individual CoTPP nanorod on a nanopore is shown in Figure 4. The defect of the gold substrate under the thin dodecanethiol SAM can be observed in both topography and the corresponding phase images (Figures 4a and 4b). In the phase image, the bare gold area of the nanopore can be discerned as a dark circle. The dimensions of the CoTPP nanorod measured $230 \mathrm{~nm}$ in length and the height is $13 \mathrm{~nm}$ (Figure 4c). 
Conductive measurements were done for the CoTPP nanorod with CP-AFM (Figure 5). Three testing points along the CoTPP nanorod were chosen for the $I-V$ curves shown in Figure 5a. Distinct differences of the $I-V$ profiles were observed for the CoTPP nanorods (Figure $5 \mathrm{~b}$ ) compared to TPP (Figure $3 \mathrm{~b}$ ). In the -2.4 to $+2.4 \mathrm{~V}$ range, semiconductive profiles are shown for each of the three $I-V$ curves shown in Figure $5 \mathrm{~b}$. The conductance measured from the positive voltage range is larger than that measured from the negative voltage range. The highest currents are -10 and $+10 \mathrm{nA}$ at -2.4 and $+2.4 \mathrm{~V}$, respectively.

To evaluate the conductive behavior of the two types of nanorods, the conductance at selected testing points on CoTPP and TPP nanorods was calculated. (The conductance was measured as the slope of the linear parts of each $I-V$ curve.) Since both CoTPP and TPP have semiconductive profiles, the conductance was categorized for the negative and positive regions of the curves (Table 1). The values of the TPP nanorods are expressed in the picosiemens range, which is a thousand times lower than that of the CoTPP nanorods. A general trend is revealed as the tip is moved along the length of the nanorods, in which the conductance increases as the probe is moved further away from the gold substrate. When one considers the larger size of the coated AFM probe, it appears that there is spatial overlap when six or more measurements are taken side-by-side. The values acquired in the positive voltage side are higher than the values obtained from the negative voltage side.

The feasibility of measuring conductance along the length of nanorods with CP-AFM was demonstrated, facilitated by the localization of the nanorods on uncovered areas of gold substrates. Arrays of of TPP and CoTPP nanorods ensured multiple, reproducible measurements with $I-V$ profiles. The properties of porphyrins at the nanoscale furnish important information for future applications.

\section{CONCLUSION}

Rod-shaped nanostructures of TPP and CoTPP were placed within nanoholes fabricated within a dodecanethiol SAM prepared by immersion particle lithography. The geometry of the nanorods enables multiple, distance-dependent measurements of nanoscale conductance using CP-AFM. The nanorods of TPP exhibit conductive profiles of an insulating material. Nanorods of CoTPP show semiconductive behavior in the longitudinal direction with the onset of the turn-on currents shifting to zero volts as the testing point approaches the end in contact with the gold surface. The strategies described herein enable measurements with a reproducible surface test platform for future studies to characterize more complex molecules.

\section{Supplementary Material}

Refer to Web version on PubMed Central for supplementary material.

\section{Acknowledgments}

The authors gratefully acknowledge financial support from the National Science Foundation Louisiana Alliance for Simulation-Guided Materials Applications (LA- SiGMA) (EPS-1003897). 


\section{References}

1. Yan S, Ding Z, Xie N, Gong H, Sun Q, Guo Y, Shan X, Meng S, Lu X. Turning on and off the Rotational Oscillation of a Single Porphine Molecule by Molecular Charge State. ACS Nano. 2012; 6:4132-4136. [PubMed: 22468858]

2. Hasobe T. Photo- and electro-functional self-assembled architectures of porphyrins. Phys Chem Chem Phys. 2012; 14:15975-15987. [PubMed: 23093225]

3. Adinehnia M, Mazur U, Hipps KW. Predicting the size distribution in crystallization of TSPP:TMPyP binary porphyrin nanostructures in a batch desupersaturation experiment. Cryst Growth Des. 2014; 14:6599-6606.

4. Guo P, Chen P, Liu M. One-dimensional porphyrin nanoassemblies assisted via graphene oxide: sheetlike functional surfactant and enhanced photocatalytic behaviors. ACS Appl Mater Interfaces. 2013; 5:5336-5345. [PubMed: 23651184]

5. Guo P, Zhao G, Chen P, Lei B, Jiang L, Zhang H, Hu W, Liu M. Porphyrin Nanoassemblies via Surfactant-Assisted Assembly and Single Nanofiber Nanoelectronic Sensors for High-Performance $\mathrm{H}_{2} \mathrm{O} 2$ Vapor Sensing. ACS Nano. 2014; 8:3402-3411. [PubMed: 24654963]

6. Medforth CJ, Wang Z, Martin KE, Song Y, Jacobsen JL, Shelnutt JA. Self-assembled porphyrin nanostructures. Chem Commun. 2009:7261-7277.

7. Arai Y, Segawa H. J-aggregation of protonated meso-tetrakis(sulfonatothienyl)porphyrin isomers: morphological selection of self-assembled nanostructures from structurally similar zwitterionic porphyrins. Chem Commun. 2010; 46:4279-4281.

8. Arai Y, Tsuzuki K, Segawa H. Homogeneously mixed porphyrin J-aggregates with rod-shaped nanostructures via zwitterionic self-assembly. Phys Chem Chem Phys. 2012; 14:1270-1276. [PubMed: 22138679]

9. Lee JH, Lee SJ. Microscopic crystalline rods from the self-assembly of mixed porphyrin building blocks. Inorg Chem Commun. 2011; 14:1014-1017.

10. Schwab AD, Smith DE, Rich CS, Young ER, Smith WF, de Paula JC. Porphyrin Nanorods. J Phys Chem B. 2003; 107:11339-11345.

11. Wan Y, Stradomska A, Fong S, Guo Z, Schaller RD, Wiederrecht GP, Knoester J, Huang L. Exciton Level Structure and Dynamics in Tubular Porphyrin Aggregates. J Phys Chem C. 2014; 118:24854-24865.

12. Rotomskis R, Augulis R, Snitka V, Valiokas R, Liedberg B. Hierarchical Structure of TPPS4 JAggregates on Substrate Revealed by Atomic Force Microscopy. J Phys Chem B. 2004; 108:28332838.

13. Jacobsen JL, Berget PE, Varela MC, Vu T, Schore NE, Martin KE, Shelnutt JA, Santos LM, Medforth CJ. Synthesis and nanostructures of 5,10,15,20-tetrakis(4-piperidyl)-porphyrin. Tetrahedron. 2013; 69:10507-10515.

14. Mongwaketsi N, Khamlich S, Klumperman B, Sparrow R, Maaza M. Synthesis and characterization of porphyrin nanotubes/rods for solar radiation harvesting and solar cells. Phys B. 2012; 407:1615-1619.

15. Vlaming SM, Augulis R, Stuart MCA, Knoester J, van Loosdrecht PHM. Exciton Spectra and the Microscopic Structure of Self-Assembled Porphyrin Nanotubes. J Phys Chem B. 2009; 113:22732283. [PubMed: 19193042]

16. Arai Y, Segawa H. Cl- complexation induced H- and J-aggregation of meso-tetrakis(4sulfonatothienyl)porphyrin diacid in aqueous solution. J Phys Chem B. 2011; 115:7773-7780. [PubMed: 21568355]

17. Li A, Zhao L, Hao J, Ma R, An Y, Shi L. Aggregation behavior of the template-removed 5,10,15,20-tetrakis(4-sulfonatophenyl)porphyrin chiral array directed by poly(ethylene glycol)block-poly(L-lysine). Langmuir. 2014; 30:4797-4805. [PubMed: 24697573]

18. Rich CC, McHale JL. Resonance Raman Spectra of Individual Excitonically Coupled Chromophore Aggregates. J Phys Chem C. 2013; 117:10856-10865.

19. Arai Y, Segawa H. Significantly enhanced adsorption of bulk self-assembling porphyrins at solid/ liquid interfaces through the self-assembly process. J Phys Chem B. 2012; 116:13575-13581. [PubMed: 23057475] 
20. Rong Y, Chen P, Liu M. Self-assembly of water-soluble TPPS in organic solvents: from nanofibers to mirror imaged chiral nanorods. Chem Commun. 2013; 49:10498-10500.

21. Reddy MH, Al-Shammari RM, Al-Attar N, Kennedy E, Rogers L, Lopez S, Senge MO, Keyes TE, Rice JH. Micro- or nanorod and nanosphere structures derived from a series of phenyl-porphyrins. Phys Chem Chem Phys. 2014; 16:4386-4393. [PubMed: 24458009]

22. Chan YH, Schuckman AE, Perez LM, Vinodu M, Drain CM, Batteas JD. Synthesis and Characterization of a Thiol-Tethered Tripyridyl Porphyrin on Au(111). J Phys Chem C. 2008; 112:6110-6118.

23. Schuckman AE, Ewers BW, Yu LH, Tomé JPC, Lisa M, Pérez LM, Drain CM, Kushmerick JG, Batteas JD. Utilizing Nearest-Neighbor Interactions To Alter Charge Transport Mechanisms in Molecular Assemblies of Porphyrins on Surfaces. J Phys Chem C. 2015; 119:13569-13579.

24. Wold DJ, Frisbie CD. Formation of Metal-Molecule-Metal Tunnel Junctions: Microcontacts to Alkanethiol Monolayers with a Conducting AFM Tip. J Am Chem Soc. 2000; 122:2970-2971.

25. Bumm LA, Arnold JJ, Dunbar TD, Allara DL, Weiss PS. Electron Transfer through Organic Molecules. J Phys Chem B. 1999; 103:8122-8127.

26. Wold DJ, Frisbie CD. Fabrication and Characterization of Metal-Molecule-Metal Junctions by Conducting Probe Atomic Force Microscopy. J Am Chem Soc. 2001; 123:5549-5556. [PubMed: 11389638]

27. Zhai X, Lee HJ, Tian T, Lee TR, Garno JC. Nanoscale lithography mediated by surface selfassembly of 16-[3,5-bis-(mercaptomethyl)phenoxy]hexadecanoic acid on $\mathrm{Au}(111)$ investigated by scanning probe microscopy. Molecules. 2014; 19:13010-13026. [PubMed: 25157466]

28. Verberne-Sutton SD, Quarels RD, Zhai X, Garno JC, Ragains JR. Application of visible light photocatalysis with particle lithography to generate polynitrophenylene nanostructures. J Am Chem Soc. 2014; 136:14438-14444. [PubMed: 25244537]

29. Tian T, LeJeune ZM, Garno JC. Directed surface assembly of 4(chloromethyl)phenyltrichlorosilane: self-polymerization within spatially confined sites of $\mathrm{Si}(111)$ viewed by atomic force microscopy. Langmuir. 2013; 29:6529-6536. [PubMed: 23642013]

30. Saner CK, Lusker KL, Lejeune ZM, Serem WK, Garno JC. Self-assembly of octadecyltrichlorosilane: Surface structures formed using different protocols of particle lithography. Beilstein J Nanotechnol. 2012; 3:114-122. [PubMed: 22428102]

31. Brownfield AL, Causey CP, Mullen TJ. Influence of Solvent on Octadecyltrichlorosilane Nanostructures Fabricated Using Particle Lithography. J Phys Chem C. 2015; 119:12455-12463.

32. Zhai X, Arachchige AMKK, Derosa P, Garno JC. Conductive-probe measurements with nanodots of free-base and metallated porphyrins. J Colloid Interface Sci. 2017; 486:38-45. [PubMed: 27693519]

33. Hegner M, Wagner P, Semenza G. Ultralarge atomically flat template-stripped Au surfaces for scanning probe microscopy. Surf Sci. 1993; 291:39-46.

34. Necas D, Klapetek P. Gwyddion: an open-source software for SPM data analysis. Cent Eur J Phys. 2012; 10:181-188.

35. Li JR, Garno JC. Nanostructures of Octadecyltrisiloxane Self-Assembled Monolayers Produced on $\mathrm{Au}(111)$ Using Particle Lithography. ACS Appl Mater Interfaces. 2009; 1:969-976. [PubMed: 20356025]

36. Li JR, Garno JC. Elucidating the role of surface hydrolysis in preparing organosilane nanostructures via particle lithography. Nano Lett. 2008; 8:1916-1922. [PubMed: 18563943] 
a

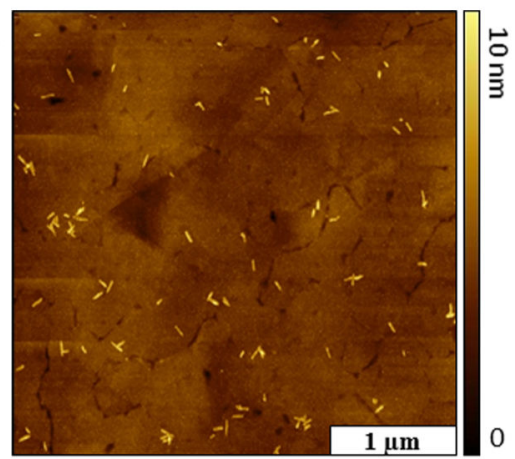

c

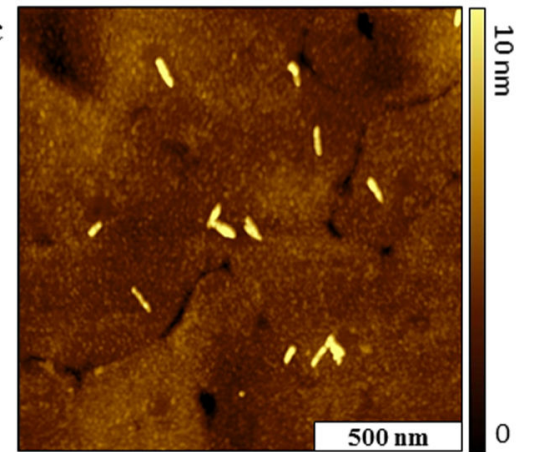

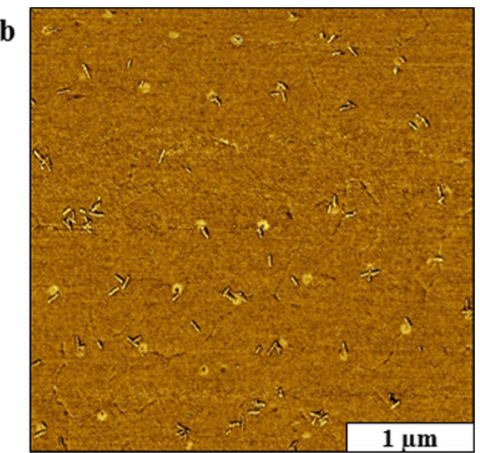

d

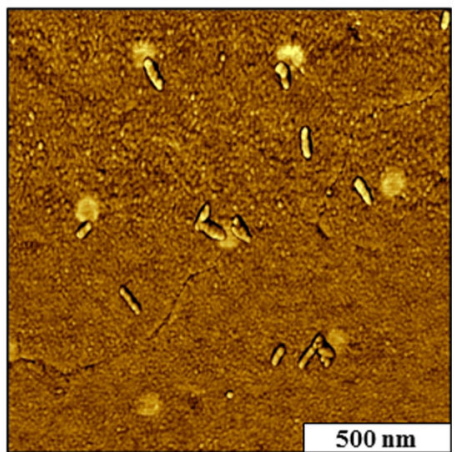

Figure 1.

Nanorods of TPP grown in nanoholes within dodecanethiol: (a) arrangement of nanorods shown with a topography image; (b) simultaneously acquired phase image; (c) localization of nanorods within nanoholes (topography view); (d) corresponding phase image. 
a
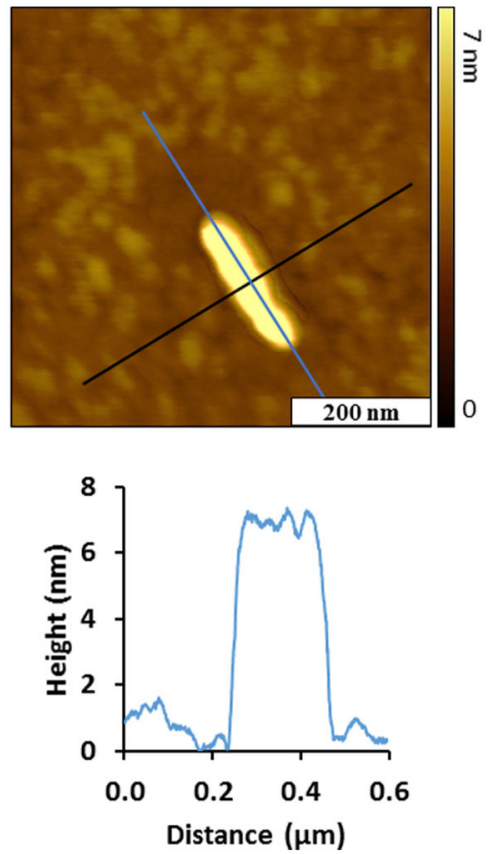

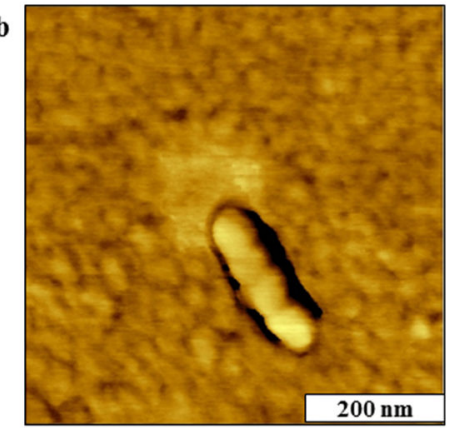

d

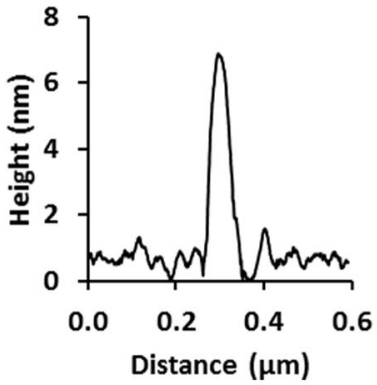

Figure 2.

Close-up of an individual TPP nanorod: (a) topography image; (b) corresponding phase image; (c) cursor profile of a longitudinal section of a nanorod; (d) cursor profile of a transverse section of a nanorod. 


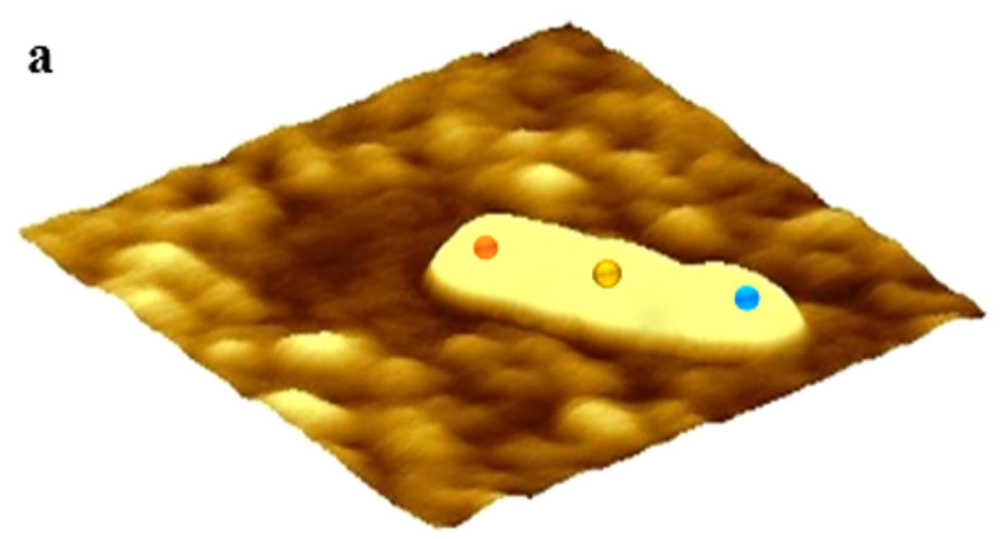

b

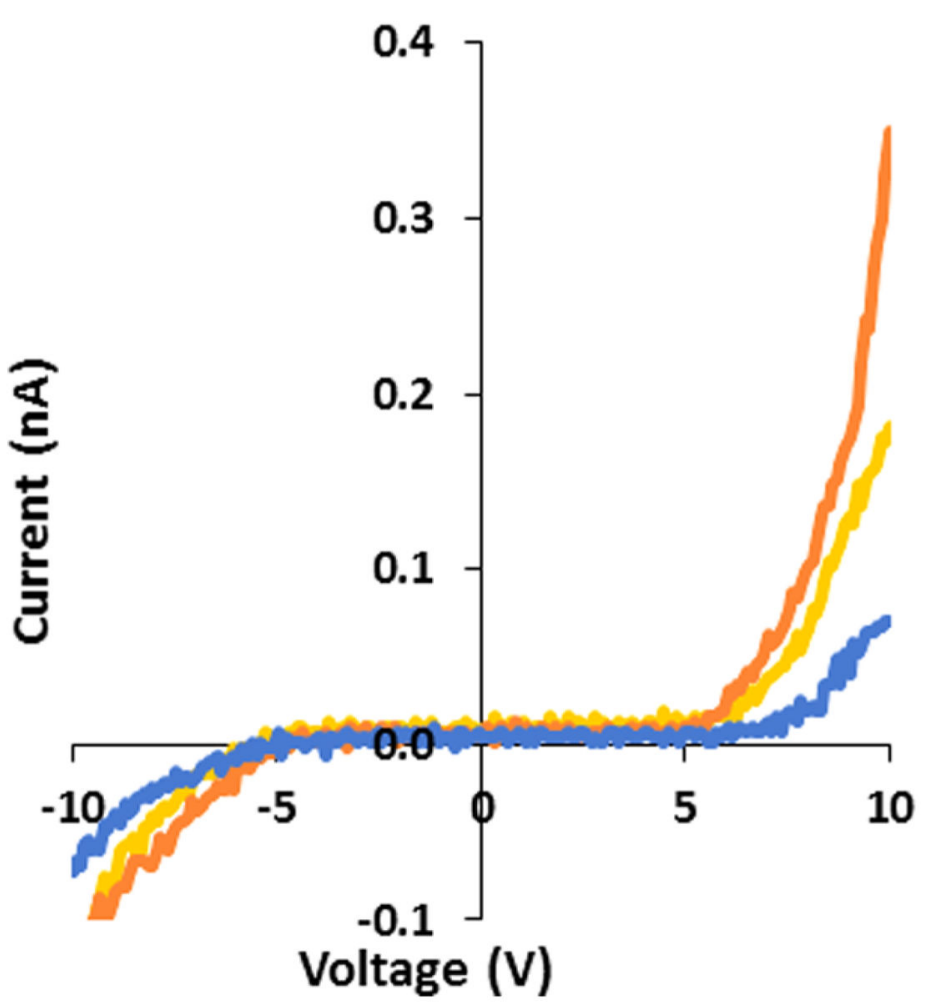

Figure 3.

Distance-dependent measurements of conductivity along a TPP nanorod. (a) A TPP nanorod localized within a nanohole. The colored dots indicate the testing points on the nanorod where $I-V$ curves were collected. (b) $I-V$ curves collected from selected locations along the TPP nanorod shown in corresponding colors. 

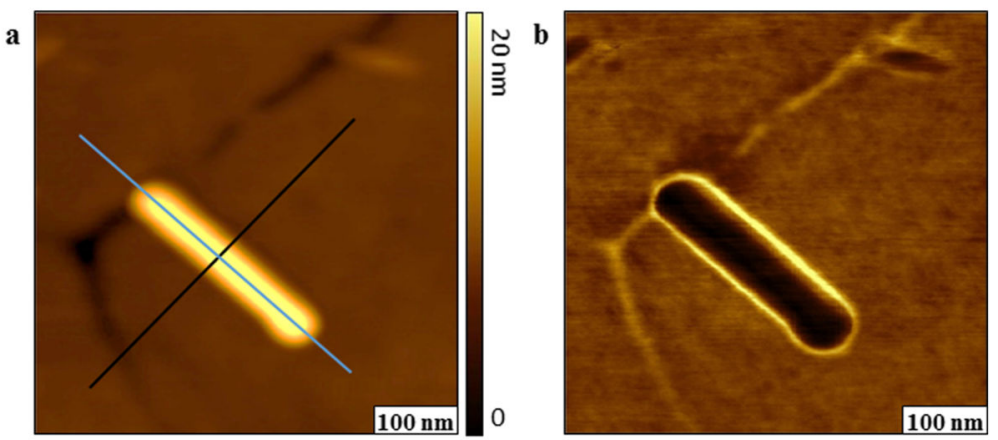

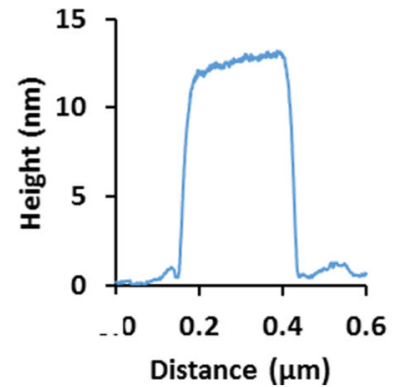

d

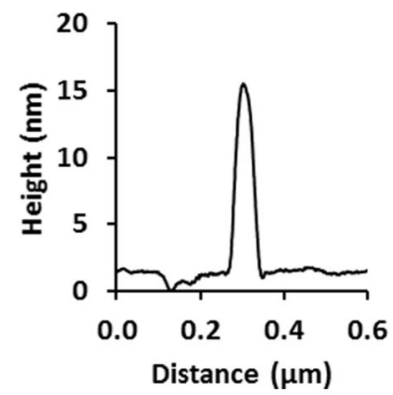

Figure 4.

Close-up view of an individual CoTPP nanorod: (a) topography image; (b) corresponding phase image; (c) cursor profile of a longitudinal section of the nanorod; (d) cursor profile of a transverse section. 

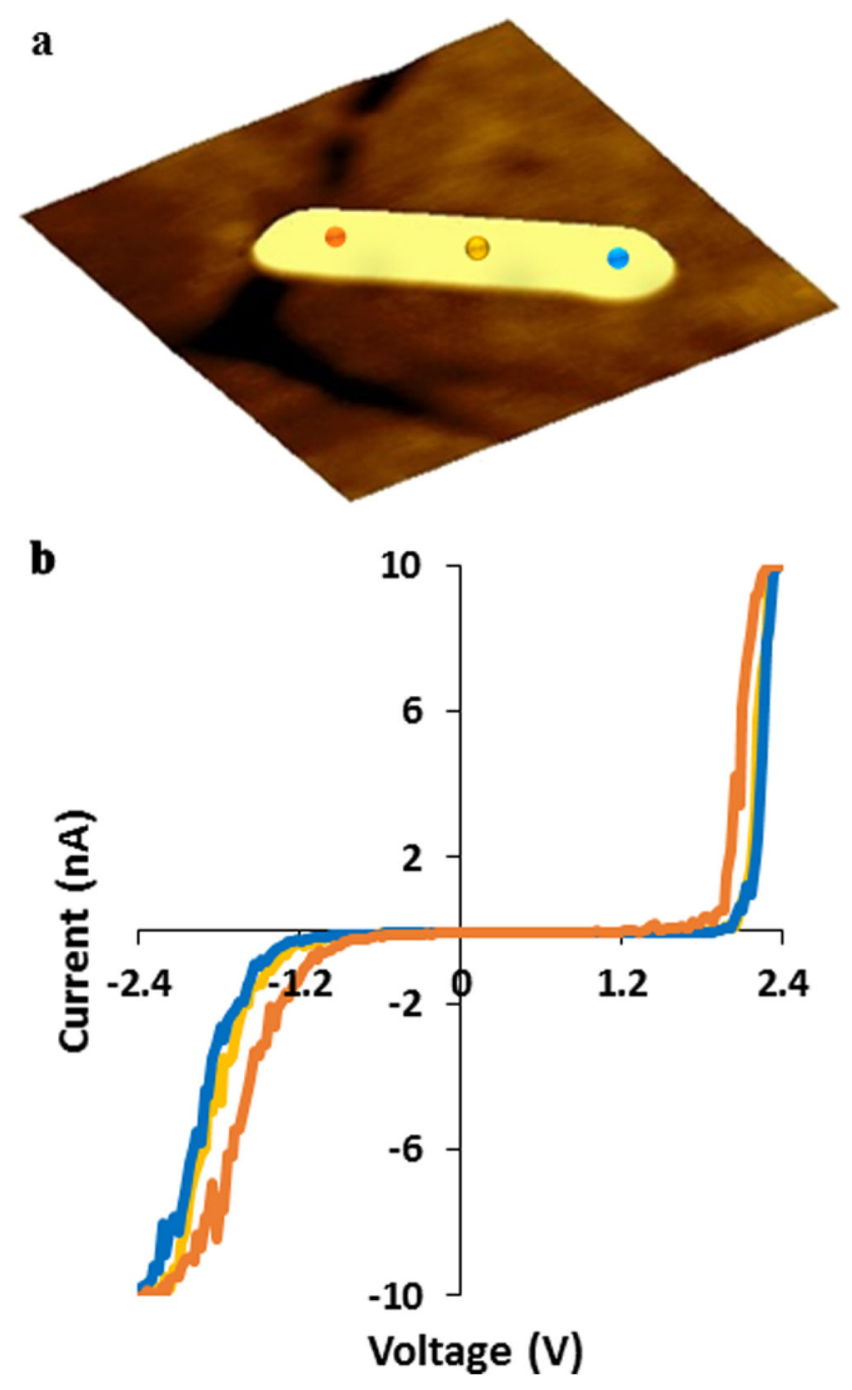

Figure 5.

Distance-dependent measurements of conductivity along a CoTPP nanorod. (a) A CoTPP nanorod localized within a nanopore. The points on the nanorod represent the sites where the $I-V$ curves were collected. (b) $I-V$ curves collected from selected locations on TPP nanorod are shown in corresponding colors. 


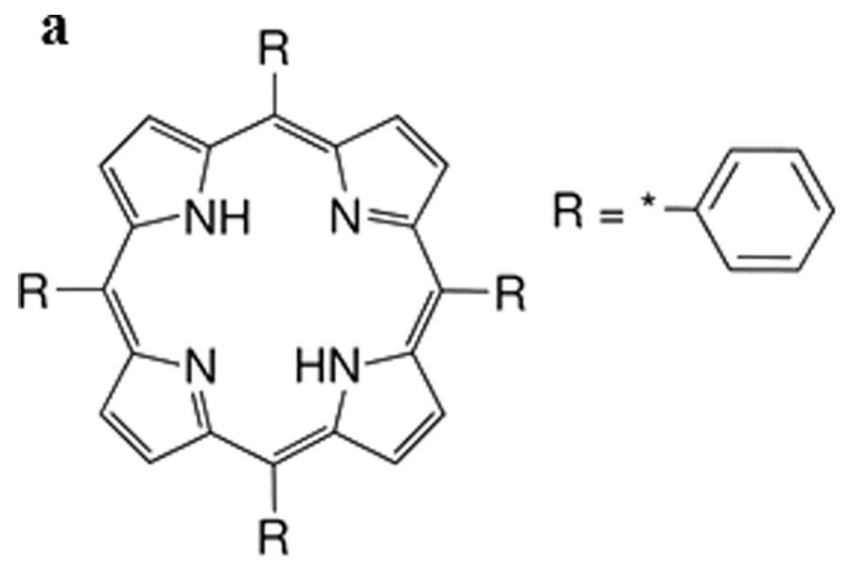

5,10,15,20-Tetraphenyl-21H,23H-porphine: TPP

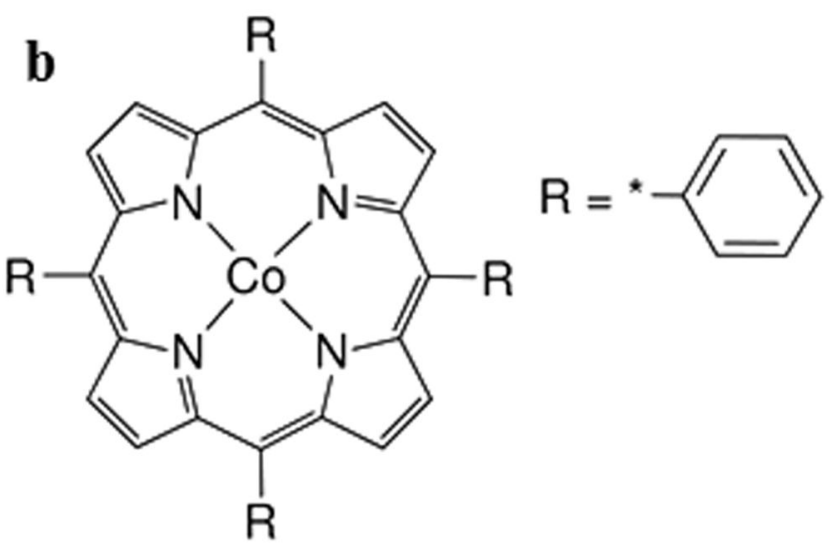

5,10,15,20-Tetraphenyl-21H,23H-porphine cobalt(II): CoTPP

Scheme 1.

Structures of 5,10,15,20-Tetraphenyl-21 $H, 23 H$-porphyrin (TPP) and 5,10,15,20-

Tetraphenyl-21 H,23H-porphyrin Cobalt(II) (CoTPP) 
Table 1

Conductance Measurements at Selected Points along TPP and CoTPP Nanorods

\begin{tabular}{cccccc}
\hline & \multicolumn{2}{c}{ TPP } & & \multicolumn{2}{c}{ CoTPP } \\
\cline { 2 - 3 } \cline { 5 - 6 } measuring point $^{\boldsymbol{a}}$ & negative bias (pS) & positive bias (pS) & & negative bias (nS) & positive bias (nS) \\
\hline 1 & 6 & 6 & 10 & 51 \\
2 & 12 & 32 & 11 & 74 \\
3 & 28 & 32 & 12 & 80 \\
4 & 14 & 51 & 14 & 32 \\
5 & 23 & 59 & 10 & 51 \\
6 & 25 & 92 & 10 & 51
\end{tabular}

a Numbers 1-6 indicate the measuring points from the end away from the gold surface (1) to the end in contact with the gold surface (6). 